\title{
Improving Spatial Reuse with Collision-Aware DCF in Mobile Ad Hoc Networks
}

\author{
Lubo Song and Chansu $\mathrm{Yu}$ \\ Department of Electrical and Computer Engineering \\ Cleveland State University \\ 2121 Euclid Avenue, Stilwell Hall 340 \\ Cleveland, $\mathrm{OH} 44115$ \\ \{l.song2,c.yu91\}@csuohio.edu
}

\begin{abstract}
According to the carrier sensing mechanism adopted by Distributed Coordination Function (DCF) in IEEE 802.11 standard, a node defers its communication if it senses the medium busy. However, this deferment might block more concurrent communications than necessary. In addition, in DCF, the time duration of deferment is not delivered to all potential interferers, particularly those in the distance. This paper proposes Collision-Aware DCF $(C A D)$ that efficiently utilizes the available channel resource along both the spatial and time dimensions. Basically, CAD embeds the spatial and time reservation requirements in the $P H Y$ header, which is transmitted at the lower data rate, so that a larger group of potential interferers become aware of the ongoing communication and thus avoid collisions. Extensive simulation based on the ns-2 has shown that CAD offers as much as $21 \%$ higher packet delivery rate and $91 \%$ lower packet delay than DCF with the CBR (constant bit rate) traffic.
\end{abstract}

\section{Introduction}

Wireless LANs (WLANs) based on IEEE 802.11 standard [1] are in great popularity in public as well as in residential areas offering new level of information accessibility and convenience of life. However, WLAN hardware as well as the underlying 802.11 standard has been originally developed for single-hop wireless communications between the access point (AP) and the end clients, which may not be appropriate for multihop communications.
This paper discusses an effort in this direction to propose an efficient medium access control (MAC) mechanism optimized for multihop environment. More specifically, this paper argues that carrier sense (CS)-based MAC algorithm such as Distributed Coordination Function (DCF) in IEEE 802.11 standard [1] does not efficiently utilize the spatial spectral resource in multihop networks. According to the DCF, a node needs to hold up its transmission if it observes the carrier signal above the pre-specified $C S$ threshold [2]. However, this may disallow more communications than necessary, which limits the reusability of the spatial spectral resource and thus the network throughput. A simplified version of this problem is a well-researched phenomenon, known as the exposed terminal problem [3].

Our goal in this paper is to develop a distributed MAC algorithm, named Collision-Aware DCF (CAD), in which each node reserves the smallest possible area just enough to protect its as well as the ongoing communications and thus helps increase the spatial spectral utilization. Contributions of this paper are four-fold:

- First, this paper highlights the importance of efficient use of spatial resource in multihop environment unlike in conventional single-hop wireless networks and proposes $\mathrm{CAD}$ where each communication expenses smallest necessary spatial area and thus overall network throughput can be increased significantly.

- Second, the CAD is a cooperative scheme in the sense that each node makes its deferment decision based not only on its own requirements but also on the collective information from nodes in the 
neighborhood. Considering the distributed nature of multihop environment, cooperation among the nodes is inevitable when it is desired to optimize the network performance. We hope this work facilitates future research on multihop networks in this direction.

- Third, another unique feature of CAD is that spatial and time reservation requirement is embedded in the PHY header rather than in the MAC header as explained in Section 4 and is shared by nodes in the neighborhood. Since the PHY header is transmitted at the lower rate, this guarantees that the information is delivered to a larger group of neighboring nodes and thus better protects the packet while efficiently utilizing the spatial resource. This way, CAD eliminates the need for virtual carrier sensing and extended interframe space (EIFS)-based spatial reservation and thus the overall algorithm becomes much simpler than DCF. To the best of the authors' knowledge, there has been no such scheme in the literature.

- Lastly, the CAD is upward compatible with technology in the sense that it can accommodate new WLAN features like transmit power and rate control. For example, different data rate derived from advanced rate control scheme such as Auto Rate Feedback (ARF) [4], Receiver-based Auto Rate (RBAR) [6], or Opportunistic Auto Rate (OAR) [7] can be used to estimate the spatial requirement and thus enhance the network performance even further. This can be similarly done for transmit power control schemes as well.

A caveat of $\mathrm{CAD}$ algorithm is that it requires incompatible changes to the IEEE 802.11 standard because of the additional fields in the PHY header. However, as discussed earlier, we believe that multihop networking demands new designs in many respects and hope this seemingly radical change would be a norm in future WLAN standards. The rest of the paper is organized as follows. Section 2 summarizes techniques that improve the spatial reusability. Section 3 presents signal propagation and reception model and analyzes the spatial and time reservation requirements for protecting a transmission in both spatial and time domains. The proposed algorithm, CAD, is introduced in Section 4. Section 5 demonstrates the performance benefits of CAD via ns- 2 simulation. Finally Section 6 draws the conclusion and describes the future work of this study.

\section{Related work}

In mobile ad hoc networks, spatial area is one of the valuable resources. Improving the spatial reusability takes more importance as wireless bandwidth is critically limited, particularly in unlicensed ISM bands. This section summarizes recent work on how this issue can be addressed using Transmit Power Control (TPC), Directional Antenna Control (DAC) and Carrier Sense Control (CSC) techniques.

\subsection{Transmit power and directional antenna control}

The idea of TPC is to apply the lowest necessary transmit power that can maintain the communication between the sender and the receiver while consuming the least energy [8-10]. For example, two control packets, Request-To-Send (RTS) and Clear-To-Send (CTS), can be exploited to detect the link quality and thus to determine the optimal power level for transmitting data packets. Data transmission uses the minimum necessary transmit power and thus saves power. However, RTS and CTS packets use the maximum power for correct operation of collision avoidance mechanism, which makes this scheme unable to enhance the spatial utilization. Nonetheless, TPC techniques have a potential to do so because smaller transmit power reduces the interference to other communications.

On the other hand, directional antenna is an explicit way of improving spatial reusability by limiting the interference range of a signal to a certain direction [11-14]. Unfortunately, directional transmissions could make the hidden terminal problem [3] more serious because more number of potential interferes become "deaf" to the ongoing communication. Multiple-Input Multiple-Output (MIMO) system [15] may alleviate this problem but dramatically increases the complexity of the transmitter and the receiver.

\subsection{Carrier sense control}

As an alternative solution, CSC has been considered recently in the literature based on the assumption that the CS threshold is tunable within the detect sensitivity of the hardware [4]. A higher CS threshold can encourage more concurrent transmissions but at the cost of more collisions. On the other hand, a lower 
CS threshold reduces the collision probability but it requires a larger spatial footprint and prevents simultaneous transmissions from occurring potentially limiting the network throughput. Obviously there is a tradeoff between high spatial reuse and increased chances of collisions [16].

Fuemmeler, et al. studied the collision prevention conditions in this context and concluded that the product of transmit power and CS threshold should be kept to be a fixed constant [17]. Zhu, et al. used an analytical model to determine the optimal CS threshold and proposed a distributed algorithm, called Adaptive Physical Carrier Sensing (APCS), that dynamically adjusts the CS threshold of an 802.11 mesh network [18, 19]. However, their analytical model does not consider the influence of MAC overhead and transmit rate, which has been addressed by Yang and Vaidya [20] and Zhai and Fang [21].

While the abovementioned schemes enhance physical carrier sensing, there also has been an active research that improves virtual carrier sensing and EIFS-based reservation mechanism. For example, in Aggressive Virtual Carrier Sensing (AVCS) [16], when a node overhears RTS or CTS but not both, it does not consider the medium as busy and is allowed to transmit its own data. $\mathrm{Li}$, et al. studied the appropriateness of EIFS and proposed Enhanced Carrier Sensing (ECS) [22]. An EIFS is used to defer transmission if an erroneous MAC packet is received according to the 802.11 DCF. However, they analyzed that the EIFS is sometimes smaller or larger than necessary, which depends on the type of the erred packets, and suggested to defer the transmission accordingly. CAD eliminates the need of EIFS because the reservation requirement is delivered to a wider group of neighbors. At the same time, CAD is superior to the abovementioned adaptive CS schemes mainly because the deferment decision is made based on the collective information from the nodes in the proximity rather than on its own.

\section{Signal propagation and reception model and reservation requirements}

\subsection{Propagation and reception model}

Signal propagation in wireless channel is affected by path loss, shadowing and multiple-path fading. This paper assumes an open area environment where path loss due to communication distance is the most important. According to the corresponding two-ray ground reflection model [23], the receive power $P_{r}(d)$ at distance $d$ is given by

$$
P_{r}(d)=P_{t} G_{t} G_{r} \frac{\left(h_{t} h_{r}\right)^{2}}{d^{4} L}
$$

where $P_{t}$ is the transmit power, $G_{t}$ and $G_{r}$ are the antenna gains of the transmitter and the receiver respectively, $h_{t}$ and $h_{r}$ are their antenna heights, and $L$ is the system loss.

Table 1. $2.4 \mathrm{GHz}$ Orinoco $11 \mathrm{~b}$ client pc card specification (Nominal output power of $15 \mathrm{dBm}$ ) [24].

\begin{tabular}{|l|c|c|c|c|}
\hline Transmit rate (Mbps) & 1 & 2 & 5.5 & 11 \\
\hline Receive sensitivity (dBm) & -94 & -91 & -87 & -82 \\
\hline Range (open area) (m) & 550 & 400 & 270 & 160 \\
\hline
\end{tabular}

To successfully receive a transmission the following two conditions have to be satisfied. First, the receiver must be within the transmission range of the sender. In other words, the receive power must be equal or larger than the receive sensitivity. Given radio hardware and transmit power, the receive sensitivity is mostly affected by the transmit rate. Table I shows their relationship of the $2.4 \mathrm{GHz}$ Orinoco $11 \mathrm{~b}$ Client PC Card [24]. Second, the receive power must be strong enough to overcome the influence of the noise and interference. This condition is described by the following signal to noise and interference ratio (SNIR) model.

$$
S N I R=\frac{P_{r}}{N_{0}+\sum I} \geq \mathrm{Z}_{0}
$$

where $N_{0}$ is the background noise, $\sum I$ is the interference from all other simultaneous transmissions, and $Z_{0}$ is the minimum required SNIR ratio, or capture ratio. The SNIR model suggests that even if more than one signal overlaps at the receiver, one of them could be survived if it is much stronger than the others. This is called the capture effect [5]. A node is staill able to transmit its data if it can capture the interference. Therefore, the node does not have to reserve a large space dictated by the CS threshold. The next subsection explains how to estimate the optimal spatial area to reserve.

\subsection{Spatial reservation requirement}

In order to make a communication successful in the presence of interference, the potential interferers in the proximity are required to defer their data transfers 
till the current communication is completed. The corresponding reservation requirement along the spatial and time dimensions will be analyzed in this and the next subsection, respectively.

Assume that a sender transmits a packet to its intended receiver with the communication distance $d$. Let $D$ be the spatial reservation requirement by the sender, i.e., the sender wishes to reserve the circular area with radius $D$ for the successful transmission of its data. Now, the purpose of this subsection is to derive the optimal (minimal) $D$ in terms of $d$ and the number of interferers, $k$. However, even though it is accomplished, how does the sender know $d$ and $k$ ? First, communication distance $d$ can be estimated based on signal strength information of the past communications with the identical receiver as discussed in Section 4.1. Second, the number of interferes, $k$, is not straightforward to estimate because a measurable quantity such as traffic intensity does not necessarily infer $k$. Fortunately, our analysis in the below shows that the minimal $D\left(D_{\min }\right)$ is practically constant irrespective the value of $k$.

According to the reception model described in the previous subsection, the SNIR at the receiver must not be less than the capture ratio $Z_{0}$. In other words,

$$
S N I R=\frac{P_{r}(d)}{N_{0}+\sum_{i=1}^{k} P_{r}\left(D_{i}\right)} \geq Z_{0}
$$

To elaborate more, we need to point out two important observations regarding $k$. First, while the interference from any node in the wireless network will affect the transmission, those from the first tier interferers, which are $D$ apart from the sender, are dominant. This is because the interference from the second tier and beyond can be safely ignored due to signal attenuation over distance. Second, since all the first tier interferers overhear and satisfy the spatial

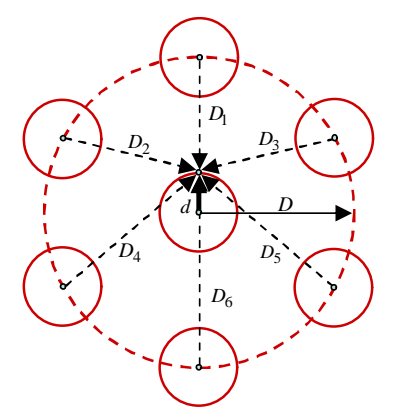

Figure 1. Worst-case interference scenario requirement $D, k$ is no more than 6 (i.e., $1 \leq k \leq 6$ ) [20] as shown in Fig. 1. Due to the space limit we only show the interference scenario with 6 interferers. Please refer to our technique report [27] for the other interference scenarios.

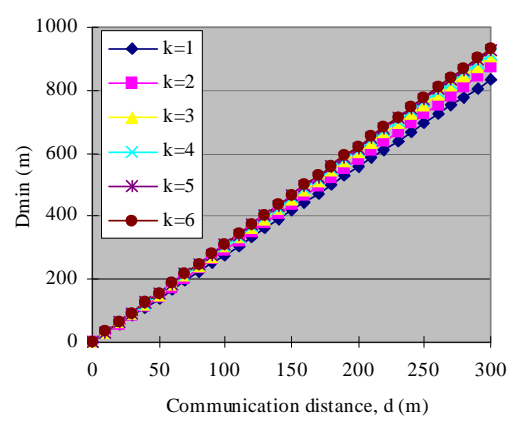

Figure 2. $D_{\min }$ versus $d$ with number of interferers $(k)$.

Now, given $d, k, Z_{0}$ and $N_{0}$, it is not difficult to find $D_{\min }$ that satisfies the inequality equation (3).

Fig. 2 plots the minimum required separating distance $\left(D_{\min }\right)$ with the effect of the communication distance $(d)$ and the number of interferes $(k)$. To our surprise, it is obvious from Fig. 2 that $d$ almost dominates the influence. For example, when $d$ is $150 \mathrm{~m}$, the variation of $D_{\min }$ with different $k$ is at most $7 \%$. This is because the signal attenuates very quickly with distance and thus the topmost interferer in Fig. 1 with the shortest distance to the receiver dominates the interference. Therefore, it is quite reasonable to assume that $k=1$ when estimating $D_{\min }$. And, when $k=1$ and $N_{0}$ is ignorable, $D_{\min }=\left(\sqrt[4]{Z_{0}}+1\right) \cdot d$ based on Equations (1) and (3).

In summary, the spatial requirement $\left(D_{\min }\right)$ can be estimated based on the communication distance $d$ and the neighbors within $D_{\min }$ from the sender are asked to hold their communications until the current data transfer is completed.

\subsection{Time reservation requirement}

Time reservation requirement in $\mathrm{CAD}$ is the time period required to protect the communication of the issuing node. This is similar to network allocation vector (NAV) specified in the 802.11 standard. CAD uses the NAV but its update is based on information included in the PLCP header rather than in MAC header in MPDU. And it protects the current frame 
immediately following the PLCP header rather than the subsequent frames. Therefore, time reservation requirement is the time duration during which the PHY payload (or MPDU itself) is transmitted. It can be calculated based on the payload length, data rate, and the type of frames. For two-way handshake model (Data and ACK frames), time reservation requirement for the Data frame should account for short interframe space (SIFS) and the ACK frame in addition to the Data frame itself. It can be similarly calculated for four-way handshake model (RTS, CTS, Data and ACK frames) but CAD does not employ RTS and CTS control frames because the hidden terminal problem is effectively addressed by the CAD algorithm.

\section{Collision-aware DCF}

This section introduces the proposed MAC mechanism, called Collision-Aware DCF (CAD). The key idea of CAD is to reserve as small spatial area and as short time period as possible so that overall spectral utilization is enhanced while protecting communications from collisions. CAD replaces physical and virtual carrier sensing as well as EIFS in 802.11 because it embeds the desired defer duration and spatial reservation in the PHY header so that even if the PHY payload is corrupted, it is quite possible for the overhearing nodes to understand the requirements and to make a proper defer decision. Section 4.1 discusses how to prepare and distribute these requirements and Section 4.2 explains how to receive and handle them.

\subsection{Preparing and distributing reservation requirements}

The spatial and time reservation requirements are denoted as REQ_SR and REQ_TR, respectively. According to the discussion in the previous section, REQ_SR is set to $P_{r}\left(D_{\min }\right)=P_{r}\left(\left(\sqrt[4]{Z_{0}}+1\right) \cdot d\right)$, the maximum interference level that the transmission can tolerate. To get the communication distance $d$, the $\mathrm{CAD}$ requires that each node maintains a neighboring list that contains the relevant signal strength information (e.g. recent received signal strength indication (RSSI)) of each neighbor. In case no signal strength information is available, the maximum communication distance corresponding to the transmit rate is assumed to estimate REQ_SR. Estimation of REQ_TR is based on frame length, frame type and

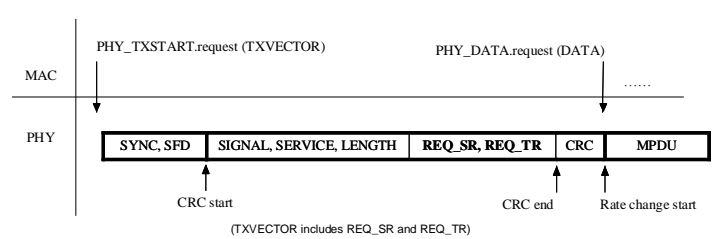

(a) Transmit procedure

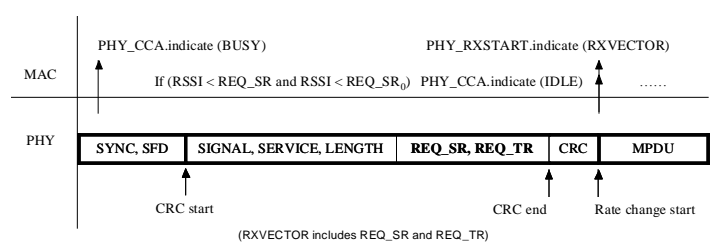

(b) Receive procedure

Figure 3. Transmit and receive procedures in the 802.11.

data rate as discussed in Section 3.3.

Now, when a backlogged node estimates REQ_SR and REQ_TR at the MAC layer, this information is added in the TXVECTOR and passed to the PHY layer along with PHY_TXSTART.request [1] as shown in Fig. 3(a). PHY layer prepares the PLCP header as in Fig. 4, where REQ_SR and REQ_TR are embedded. It then transmits the frame according to the transmit procedure specified in the 802.11 standard [1], which not only delivers the header to the receiver but also distributes the reservation requirements to the neighboring potential interferers so that they can optimally decide whether or not to comply. Data rate used for MPDU transmission is indicated by the 8-bit SIGNAL field and the MPDU is transmitted upon PHY_DATA.request as in Fig. 3(a).

Embedding the reservation requirement of a transmission in the PLCP header has two benefits: First, a neighbor can immediately determine if it is the potential interferer of the ongoing communication when it receives the PLCP header. Second, since the PLCP header is transmitted at the lowest data rate, it reaches nodes in farther distance as in Table I. While neighbors in direct transmit range would not cause any trouble because they hear the ongoing communication anyway, those in farther distance are the most critical potential interferers. In 802.11, they

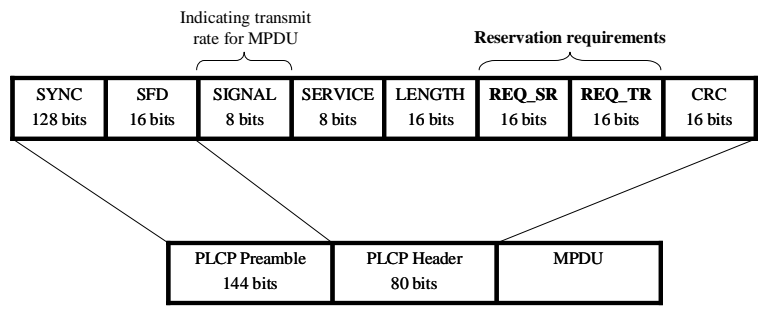

Figure 4: PLCP frame format. 
are inhibited partly by RTS/CTS exchange and partly by EIFS as discussed earlier. However, CAD embeds the requirements in the PLCP header so that they are delivered to a larger group of neighbors in a simpler manner.

\subsection{Receiving and handling reservation requirements}

Fig. 3(b) shows the PLCP receive procedure. Upon detecting a coming signal, PHY_CCA.indicate (BUSY) will be issued to the MAC layer if the signal strength is higher than CS threshold. Then PHY will begin searching for Start Frame Delimiter (SFD) and start to receive a PLCP header. If a PLCP header is successfully received (CRC check passes), the PHY_RXSTART (RXVECTOR) will be issued to the MAC layer according to the 802.11. The RXVECTOR contains the information of SIGNAL field, SERVICE field, LENGTH field, RSSI, signal quality, and antenna used for receive.

In CAD, RXVECTOR includes REQ_SR and REQ_TR in addition to the information mentioned above. When a backlogged node receives a PLCP header successfully, it has to make to two decisions: Whether its communication is successful if it transmits concurrently with the current data transfer. And, whether the current communication is successful if it transmits. For the former question, the node compares the RSSI of the incoming signal with its own spatial requirement, denoted as REQ_SR $R_{0}$. In other words, the node defers if $\mathrm{RSSI} \geq \mathrm{REQ} \mathrm{SR}_{0}$ because the strength of the incoming signal exceeds the maximum interference level that its outgoing transmission can tolerate. For the latter, the node compares the RSSI of the incoming signal with REQ_SR of the current transmission. I.e., the node defers if $R S S I \geq R E Q$ SR because the current communication would fail if the node transmits. This is based on the assumption that the link is symmetric; The RSSI of the incoming signal is equal to the RSSI (interference) that the node would cause to the ongoing transmission.

In summary, if $\mathrm{RSSI} \geq \mathrm{REQ} \mathrm{SR}_{0}$ or $\mathrm{RSSI} \geq \mathrm{REQ}$ _SR, the medium is considered busy and the node holds up its transmission. In this case, PHY will continue to receive MPDU but NAV is set to a new value REQ_TR obtained from the incoming PLCP header. On the other hand, if $R S S I<R E Q \_S R_{0}$ and RSSI $<$ REQ_SR, the medium is considered idle. In this case, PHY will issue PHY_CCA.indicate(IDLE) to the MAC layer so that the node can transmit its frame even though there is an ongoing communication. Therefore, CAD encourages more concurrent communications as long as they do not interfere with each other and thus increases the network throughput.

\section{Performance evaluation}

In order to evaluate the performance improvement of CAD, this section compares CAD with DCF2 (DCF without RTS/CTS exchange) and DCF4 (DCF with RTS/CTS exchange) based on $n s-2$ [25]. Both DCF2 and DCF4 are evaluated in this paper for fair comparison because the proposed CAD does not employ the RTS/CTS handshake. As discussed later in this section, DCF2 shows better performance than DCF4, which is counter-intuitive but has been predicted by a number of researchers in the literature [26]. This is mainly due to the overly sensitive CS threshold. In other words, the RTS/CTS handshake simply adds MAC control overhead with little gain in the current setting of the radio parameters.

\subsection{Simulation environment}

Our performance study is based on $n s-2$ simulation of 100 mobile nodes that are distributed in a $300 \times 1500$ $\mathrm{m}^{2}$ area. The movement of the nodes is described by the random waypoint mobility model with the maximum speed of $5 \mathrm{~m} / \mathrm{s}$ and with the pause time of 100s. 10 50 CBR (constant bit rate) traffic is used to simulate the network traffic. The simulation time is 900s and each simulation scenario is tested with five runs to obtain the average performance measures.

$N s$-2 network simulator simulates the aforementioned node movement, traffic generation, radio interference, and DCF protocol. Ad-hoc Ondemand Distance Vector (AODV) routing protocol is used in our study to find the routing path. The radio propagation model used is two-ray ground propagation model expressed by Equation (1). Based on the radio parameters of the $2.4 \mathrm{GHz}$ Orinoco $11 \mathrm{~b}$ Client PC Card [24], receive sensitivity is set to $94 \mathrm{dBm}$ and $-87 \mathrm{dBm}$ for data rates of $1 \mathrm{Mbps}$ and $5.5 \mathrm{Mbps}$, respectively. This translates to the transmit range of $550 \mathrm{~m}$ and $270 \mathrm{~m}$ with the transmit power of $15 \mathrm{dBm}$ as shown in Table 1. Default CS threshold used by DCF is set as $-94 \mathrm{dBm}(550 \mathrm{~m})$. Capture ratio of $10 \mathrm{~dB}$ is used in our performance study. 


\subsection{Simulation results and discussion}

This subsection presents simulations results comparing the performance of the proposed CAD with DCF2 and DCF4. Packet delivery ratio (PDR) and packet delay are used as primary performance metrics in this study. Fig. 5 shows them with respect to mobility. Note that pause time of 900 s translates to a static network environment where nodes do not move because the simulation is time is 900 s. Pause time of 0s corresponds to a constant moving scenario. $30 \mathrm{CBR}$ connections are simulated where source and destination nodes are chosen randomly among the 100 mobile nodes. Each traffic source generates five 1024byte packets every second. As shown in the figure, CAD significantly outperforms DCF2 and DCF4. For example, when the pause time is 300 s, CAD achieves $20 \%$ and $44 \%$ higher PDR than DCF2 and DCF4, respectively. Also, CAD shows $77 \%$ and $82 \%$ reduction in packet delay.

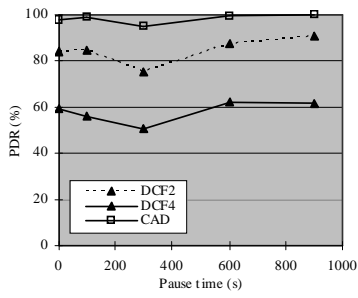

(a) PDR

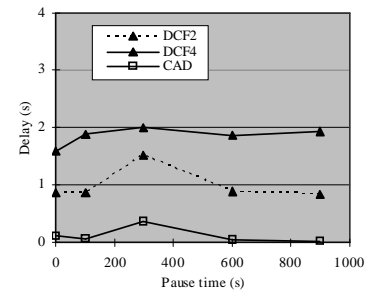

(b) Delay
Figure 5. End-to-end performance comparison with mobility.

The dramatic performance improvement of CAD over DCF2 and DCF4 is attributed to higher concurrency and its better prediction capability of collisions. In DCF, nodes make transmission decisions depending on the carrier signal and the predetermined CS threshold. However, in CAD, nodes make decisions based on information from their neighbors as well as the carrier signal strength and thus result in a lower collision probability. This is proven via simulation as shown in Fig. 6(a). It shows the number of collided packets (unicast packets such as MAC layer and routing layer control packets and data packets) collected during the simulation period of 900s. CAD shows as much as 3.2 and 5.6 times less number of packet collisions than DCF2 and DCF4, respectively. Comparing DCF2 and DCF4, majority of collisions is due to data packets in DCF2 while that in DCF4 is due to RTS.

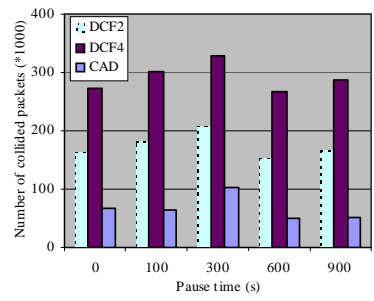

(a) Number of collisions

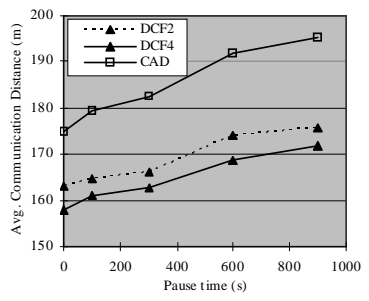

(b) Avg. comm. distance
Figure 6. Per-hop performance comparison with mobility.

In Fig. 6(b), CAD shows longer average per-hop communication distance. We'll explain the reasons as well as its implications in the below. In both DCF and CAD, short-hop communications are successful with a high probability because they are stronger to interference due to the capture effect as discussed earlier in Section 3. On the other hand, longer-hop communications are easily subjective to interference and result in collisions. Since CAD exploits the information about ongoing communication to avoid collisions, longer-hop communications in CAD are safer than in DCF2 or DCF4. Therefore, average communication distance of CAD is longer as shown in Fig. 6(b). Now, longer-hop distance implies smaller hop count for a given source-destination pair and thus less number of per-hop communications. This is another benefit of $\mathrm{CAD}$ and can lead to higher network throughput.

We also investigated the affects of traffic intensity and transmit rates. CAD still outperforms DCF. Due to space limit we do not present the results here. For the details please refer to our technique report [27].

\section{Conclusions and future work}

This paper proposes Collision-Aware DCF (CAD) mechanism that encourages more concurrent transmissions but at the same time avoids collisions more efficiently. While the DCF avoids collisions based on a pre-determined carrier sense threshold (physical carrier sense) and advertise of the communication duration embedded in the MAC header (virtual carrier sense), both methods often fail to achieve the maximum achievable performance, particularly in multihop network environment. In $\mathrm{CAD}$, each node estimates the range that it wishes to reserve for its data transfer (spatial reservation requirement) and the time duration (time reservation requirement) based on the communication distance, 
transmit rate, packet type and size. And, they are embedded in the PLCP header of the transmitted packet so that a larger group of potential interferers become aware of it and ahead of time. Our simulation study based on ns- 2 shows that CAD significantly improves the network performance in terms of packet delivery ratio and packet delay. It is observed that the benefit of CAD comes from less number of collisions, which in fact was the original goal of the CAD mechanism.

CAD is designed to be compatible with TPC and TRC capability in the sense that estimation of the spatial reservation requirement can easily accommodate the transmit power and transmit rate information. This issue needs further study and is remained as a future work. In this paper we only verified the benefits of CAD with two transmit rates (1Mbps and 5.5Mbps). The further investigation of CAD with adaptive transmit rate scheme proposed in $[4,6,7]$ is another future work.

\section{References}

[1] IEEE Std 802.11-1999, Local and Metropolitan Area Network, Specific Requirements, Part 11: Wireless LAN Medium Access Control (MAC) and Physical Layer (PHY) Specifications, http://standards.ieee.org/ getieee802/download/802.11-1999.pdf.

[2] A. Umar, "Wireless LANs-802.11 and Mobile Ad Hoc Networks," Ch. 6, Mobile Computing and Wireless Communications, NGE Solutions, Inc., 2004.

[3] J. Schiller, "Wireless LAN," Ch. 7, Mobile Communications, Addison-Wesley, Harlow, UK, 2000.

[4] A. Kamerman and L. Monteban, "WaveLAN-II: A High-Performance Wireless LAN for the Unlicensed Band," Bell Labs Technical Journal, pp. 118-133, Summer 1997.

[5] M. Zorzi, and R. Rao, "Capture and retransmission control in mobile radio," IEEE Journal on Selected Areas in Communications, 12(8), pp. 1289-1298, 1994.

[6] G. Holland, N. Vaidya, and P. Bahl, "A Rate-Adaptive MAC Protocol for Multi-Hop Wireless Networks," ACM Mobicom, 2001.

[7] B. Sadeghi, V. Kanodia, A. Sabharwal, and E. Knightly, "OAR: An Opportunistic Autorate Media Access Protocol for Ad Hoc Networks," ACM MobiCom, 2002.

[8] J. P. Monks, V. Bharaghavan and W. W. Hwu, "A Power Controlled Multiple Access Protocol for Wireless Packet Networks," IEEE INFOCOM, 2001

[9] J. Monks, J.-P. Ebert, W.-M. W. Hwu and A. Wolisz, "Energy saving and capacity improvement potential of power control in multi-hop wireless networks," Computer Networks, pp. 313-330, 41 (2003), 2003.

[10] C. Yu, K. G. Shin, and B. Lee, "Power-Stepped
Protocol: Enhancing Spatial Utilization in a Clustered Mobile Ad Hoc Network," IEEE Journal of Selected Areas in Communications, 22(7), pp. 1322-1334, 2004.

[11] Y. B. Ko, V. Shankarkumar and N. H. Vaidya, "Medium Access control protocols using directional antennas in ad hoc networks," IEEE INFOCOM, 2000.

[12] M. Takai, J. Martin, R. Bagrodia, and A. Ren, "Directional virtual carrier sensing for directional antennas in mobile ad hoc networks," ACM MobiHOC, 2002.

[13] Y. Wang and J. J. Garcia-Luna-Aceves, "Spatial Reuse and Collision Avoidance in Ad Hoc Networks with Directional Antennas," IEEE GLOBECOM, 2002.

[14] T. Korakis, G. Jakllari, and L. Tassiulas, "A MAC protocol for full exploitation of Directional Antennas in Ad-hoc Wireless Networks," ACM MobiHOC, 2003.

[15] J. S. Park, A. Nandan, M. Gerla and H. Lee, "SPACEMAC Enabling Spatial Reuse Using MIMO Channelaware MAC," IEEE ICC, 2005.

[16] F. Ye, S. Yi, and B. Sikdar, "Improving Spatial reuse of IEEE 802.11 Based Ad Hoc Networks," IEEE GLOBECOM, 2003.

[17] J. Fuemmeler, N. Vaidya, and V. Veeravalli, "Selecting Transmit Powers and Carrier Sense Thresholds for CSMA Protocols," Technique report, University of Illinois at Urbana-Champaign, October 2004.

[18] J. Zhu, X. Guo, L. L. Yang, and W. S. Conner, "Leveraging Spatial Reuse in 802.11 Mesh Networks with Enhanced Physical Carrier Sensing," IEEE ICC, pp. 4004-4011, 2004.

[19] J. Zhu, X. Guo, L. L. Yang, W. S. Conner, S. Roy and M. M. Hazra, "Adapting physical carrier sensing to maximize spatial reuse in 802.11 mesh networks," Wireless Communications and Mobile Computing, (4), pp. 933-946, 2004.

[20] X. Yang and N. Vaidya, "On the Physical Carrier Sense in Wireless Ad Hoc Networks," IEEE INFOCOM, 2005.

[21] H. Zhai and Y. Fang, "Physical Carrier Sensing and Spatial Reuse in Multirate and Multihop Wireless Ad Hoc Networks," IEEE INFOCOM, 2006 (to appear).

[22] Z. F. Li, S. Nandi, and A. K. Gupta, "Improving MAC Performance in Wireless Ad Hoc Networks using Enhanced Carrier Sensing (ECS)," In IFIP Networking, 2004.

[23] T. Rappaport, Wireless communications: principles and practice. $2^{\text {nd }}$ Edition, Prentice Hall Communications Engineering and Emerging Technologies Series. Prentice Hall, 2002.

[24] Orinoco 11b Client PC Card Specification. Available from http://www.proxim.com/learn/library/datasheets/ 11bpccard.pdf, 2005.

[25] Network Simulator ns-2, http://www.isi.edu/nsnam/ns/.

[26] C. Yu, K. G. Shin, and L. Song, "Link-Layer Salvaging for Making Progress in Mobile Ad Hoc Networks," ACM MobiHoc, 2005.

[27] L. Song, and C. Yu, "Collision-Aware DCF," Technique Report of Cleveland State University, 2006. 